\title{
Note on translated sum on primitive sequences
}

\author{
Ilias Laib \\ ENSTP, Garidi Kouba, 16051, Algiers, \\ and Laboratory of Equations with Partial Non-Linear Derivatives, \\ ENS Vieux Kouba, Algiers, Algeria \\ e-mail: laib23@yahoo.fr
}

Received: 21 September 2020

Revised: 21 July 2021

Accepted: 27 August 2021

Abstract: In this note, we construct a new set $S$ of primitive sets such that for any real number $x \geq 60$ we get:

$$
\sum_{a \in \mathcal{A}} \frac{1}{a(\log a+x)}>\sum_{p \in \mathcal{P}} \frac{1}{p(\log p+x)}, \mathcal{A} \in \boldsymbol{S},
$$

where $\mathcal{P}$ denotes the set of prime numbers.

Keywords: Primitive sequences, Erdős's conjecture, Prime numbers, Integer sequences.

2020 Mathematics Subject Classification: 11B05, 11Y55, 11 L20.

\section{Introduction}

A sequence $\mathcal{A}$ of strictly positive integers is said to be primitive if none of its elements divide another. From the sequence of prime numbers $\mathcal{P}=\left(p_{n}\right)_{n \geq 1}$ we can construct an infinite collection of primitive sequences. Indeed, all the following sequences

$$
\begin{aligned}
\mathcal{A}_{d}^{k} & =\left\{p_{1}^{\alpha_{1}} p_{2}^{\alpha_{2}} \cdots p_{k}^{\alpha_{k}} \mid \alpha_{1}, \ldots, \alpha_{k}, d \in \mathbb{N}, \alpha_{1}+\cdots+\alpha_{k}=d, d \geq 1\right\}, \\
\mathcal{A}^{k} & =\left\{p_{n} \in \mathcal{P} \mid n>k\right\}, \mathcal{B}_{d}^{k}=\mathcal{A}_{d}^{k} \cup \mathcal{A}^{k},
\end{aligned}
$$

are primitive. According to the prime number theorem, the $n$-th prime number $p_{n}$ is asymptotically equal to $n \log n$; this ensures the convergence of the series

$$
S(\mathcal{P})=\sum_{p \in \mathcal{P}} \frac{1}{p \log p} .
$$

A computation for $S(\mathcal{P})$ was obtained in [2] by Cohen as:

$$
S(\mathcal{P})=1.63661632335126086856965800392186367118159707613129 \ldots .
$$


Throughout this paper we assume that $\mathcal{A} \notin\{\varnothing,\{1\}\}$. In [3], Erdős proved that the series $S(\mathcal{A})$ converges for any primitive sequence $\mathcal{A}$ and in [4], Erdős asked if it is true that $S(\mathcal{A}) \leq$ $S(\mathcal{P})$ for any primitive sequence $\mathcal{A}$. In [5], Erdős and Zhang showed that $S(\mathcal{A}) \leq 1.84$ for any primitive sequence $\mathcal{A}$, and in [1], Clark improved this result $S(\mathcal{A}) \leq e^{\gamma}$ (where $\gamma$ is the Euler constant) in the special case when $\mathcal{A}$ is a primitive set of composite numbers. Several years later in [8], Lichtman and Pomerance proved that $S(\mathcal{A})<e^{\gamma} \simeq 1.781$. Moreover, in [5], Erdôs conjectured that $S(\mathcal{A}) \leq S(\mathcal{P})$ for any primitive sequence $\mathcal{A}$, then in [11,12], Zhang proved this conjecture in some special cases of primitive sequences. In [7], the authors show that the analogue of the Erdôs conjecture, which was studied by Farhi in [6], is not satisfied for the translated sums of the form:

$$
S(\mathcal{A}, x)=\sum_{a \in \mathcal{A}} \frac{1}{a(\log a+x)} \text { for } x \geq 81 .
$$

Later in [9], the authors show that for $x$ large enough, there exists a primitive sequence $\mathcal{A}$, such that $S(\mathcal{A}, x)>>S(\mathcal{P}, x)$. In this article, we improve the result of [7] as follows:

Theorem 1.1. Let $k_{0}=130947$ and $x_{0}=60$. For any integer $k \geq k_{0}$, we have:

$$
S\left(\mathcal{B}_{2}^{k}, x\right)>S(\mathcal{P}, x) \text { for } x \geq x_{0} .
$$

To prove this theorem, we need the following lemmas.

\section{Lemmas}

Lemma 2.1. ([10]) For any real number $x>1$, we have:

$$
\sum_{p \in \mathcal{P}, p \leq x} \frac{1}{p}>\log \log x+\beta-\frac{1}{2 \log ^{2} x} \text { where } \beta=0.261497212847643 \ldots
$$

Lemma 2.2. Let $x_{1}, x_{1}, \ldots, x_{n}$ be non-zero real numbers, then we have:

$$
\sum_{1 \leq i \leq j \leq n} x_{i} x_{j}=\frac{1}{2}\left(\left(\sum_{1 \leq i \leq n} x_{i}\right)^{2}+\sum_{1 \leq i \leq n} x_{i}^{2}\right) .
$$

Proof. By induction.

Lemma 2.3. ([7]) For all integer $k \geq 1$ and all integer $d \geq 2$, we have the (disjoint) union

$$
\mathcal{A}_{d}^{k+1}=\mathcal{A}_{d}^{k} \cup\left\{a p_{k+1} \mid a \in \mathcal{A}_{d-1}^{k+1}\right\} .
$$

Lemma 2.4. Let $k^{\prime}=58$. For any real number $x>0$, the sequence $\left(\mathcal{S}\left(\mathcal{B}_{2}^{k}, x\right)\right)_{k \geq k^{\prime}}$ is strictly increasing.

Proof. According to Lemma 2.2, for any integer $k \geq 1$ we have:

$$
\sum_{a \in \mathcal{A}_{2}^{k}} \frac{1}{a}=\sum_{1 \leq i \leq j \leq k} \frac{1}{p_{i} p_{j}}=\frac{1}{2}\left(\left(\sum_{1 \leq i \leq k} \frac{1}{p_{i}}\right)^{2}+\sum_{1 \leq i \leq k}\left(\frac{1}{p_{i}}\right)^{2}\right) .
$$


For $1 \leq i \leq k$, we have $p_{i} \leq p_{k}$, so

$$
\begin{aligned}
\sum_{a \in \mathcal{A}_{2}^{k}} \frac{1}{a} & \geq \frac{1}{2}\left(\left(\sum_{1 \leq i \leq k} \frac{1}{p_{i}}\right)^{2}+\frac{1}{p_{k}} \sum_{1 \leq i \leq k} \frac{1}{p_{i}}\right) \\
& \geq \frac{1}{2}\left(\sum_{1 \leq i \leq k} \frac{1}{p_{i}}+\frac{1}{p_{k}}\right) \sum_{1 \leq i \leq k} \frac{1}{p_{i}}
\end{aligned}
$$

According to Lemma 2.3, we have:

$$
\mathcal{B}_{2}^{k+1}=\mathcal{A}_{2}^{k+1} \cup \mathcal{A}^{k+1}=\mathcal{A}_{2}^{k} \cup\left\{a p_{k+1} \mid a \in \mathcal{A}_{1}^{k+1}\right\} \cup \mathcal{A}^{k+1},
$$

so

$$
S\left(\mathcal{B}_{2}^{k+1}, x\right)-S\left(\mathcal{B}_{2}^{k}, x\right)=\frac{1}{p_{k+1}}\left(S\left(\mathcal{A}_{1}^{k+1}, \log p_{k+1}+x\right)-\frac{1}{\log p_{k+1}+x}\right) .
$$

Knowing that $p_{k+1}$ is the largest element of $\mathcal{A}_{1}^{k+1}$, so we have:

$$
S\left(\mathcal{A}_{1}^{k+1}, \log p_{k+1}+x\right)=\sum_{a \in \mathcal{A}_{1}^{k+1}} \frac{1}{a\left(\log a+\log p_{k+1}+x\right)} \geq \frac{1}{2 \log p_{k+1}+x} \sum_{n=1}^{k+1} \frac{1}{p_{n}} .
$$

A computer calculation gives

$$
\sum_{n=1}^{k+1} \frac{1}{p_{n}} \geq \sum_{n=1}^{k^{\prime}+1} \frac{1}{p_{n}}=2.0023 \ldots>2 \text { for } k \geq k^{\prime}
$$

therefore,

$$
\begin{aligned}
S\left(\mathcal{A}_{1}^{k+1}, \log p_{k+1}+x\right)-\frac{1}{\log p_{k+1}+x} & >\frac{2}{2 \log p_{k+1}+x}-\frac{1}{\log p_{k+1}+x} \\
& =\frac{x}{\left(2 \log p_{k+1}+x\right)\left(\log p_{k+1}+x\right)}>0,
\end{aligned}
$$

so $S\left(\mathcal{B}_{2}^{k+1}, x\right)-S\left(\mathcal{B}_{2}^{k}, x\right)>0$.

\section{Proof of Theorem 1.1}

For any integer $k \geq 1$, the number $p_{k}^{2}$ is the largest element of the primitive sequence $\mathcal{A}_{2}^{k}$, so for any $a \in \mathcal{A}_{2}^{k}$ we have $\log a \leq 2 \log p_{k}$. Then for any $x>0$, we have:

$$
\begin{aligned}
\sum_{a \in \mathcal{B}_{2}^{k}} \frac{1}{a(\log a+x)} & =\sum_{a \in \mathcal{A}_{2}^{k} \cup \mathcal{A}^{k}} \frac{1}{a(\log a+x)}=\sum_{a \in \mathcal{A}_{2}^{k}} \frac{1}{a(\log a+x)}+\sum_{a \in \mathcal{A}^{k}} \frac{1}{a(\log a+x)} \\
& \geq \frac{1}{2 \log p_{k}+x} \sum_{a \in \mathcal{A}_{2}^{k}} \frac{1}{a}+\sum_{n>k} \frac{1}{p_{n}\left(\log p_{n}+x\right)} .
\end{aligned}
$$

From (1) and Lemma 2.1, we obtain:

$$
\sum_{a \in \mathcal{A}_{2}^{k}} \frac{1}{a}>\frac{1}{2}(x+\log 2)\left(\log \log p_{k}+\beta-\frac{1}{2 \log ^{2} p_{k}}+\frac{1}{p_{k}}\right) \sum_{i=1}^{k} \frac{1}{(x+\log 2) p_{i}},
$$


SO

$$
\begin{aligned}
\sum_{a \in \mathcal{B}_{2}^{k}} \frac{1}{a(\log a+x)} & \geq \frac{(x+\log 2)}{2\left(2 \log p_{k}+x\right)} \sum_{a \in \mathcal{A}_{2}^{k}} \frac{1}{a}+\sum_{n>k} \frac{1}{p_{n}\left(\log p_{n}+x\right)} \\
& >\frac{(x+\log 2)\left(\log \log p_{k}+\beta-\frac{1}{2 \log ^{2} p_{k}}+\frac{1}{p_{k}}\right)}{2\left(2 \log p_{k}+x\right)} \sum_{n=1}^{k} \frac{1}{p_{n}\left(\log p_{n}+x\right)}+\sum_{n>k} \frac{1}{p_{n}\left(\log p_{n}+x\right)} .
\end{aligned}
$$

To obtain the inequality required in Theorem 1.1 , it is necessary to choose $k$ and $x$ so that

$$
\frac{(x+\log 2)\left(\log \log p_{k}+\beta-\frac{1}{2 \log ^{2} p_{k}}+\frac{1}{p_{k}}\right)}{2\left(2 \log p_{k}+x\right)}>1 .
$$

It is clear that the function

$$
x \longmapsto h_{k}(x)=\frac{\left(\log \log p_{k}+\beta-\frac{1}{2 \log ^{2} p_{k}}+\frac{1}{p_{k}}\right)(x+\log 2)}{2\left(2 \log p_{k}+x\right)} \text { for } k>1,
$$

is strictly increasing for $x \in \mathbb{R}^{*}$. Let choose $k$ and $x_{0}$ such that $x_{0}$ is an integer and the minimum for which the above inequality holds. Then

$$
\frac{\log \log p_{k}+\beta-\frac{1}{2 \log ^{2} p_{k}}+\frac{1}{p_{k}}-2}{4 \log p_{k}-\log 2\left(\log \log p_{k}+\beta-\frac{1}{2 \log ^{2} p_{k}}+\frac{1}{p_{k}}\right)}>\frac{1}{x_{0}} .
$$

Since $x_{0}>0$, so for the $k$ we choose, we must verify that:

$$
\log \log p_{k}-\frac{1}{2 \log ^{2} p_{k}}+\frac{1}{p_{k}}-2+\beta>0 .
$$

The growth of the function $t \rightarrow g(t)=\log \log t-\frac{1}{2 \log ^{2} t}+\frac{1}{t}-2+\beta$ for $t>1$, gives us $p_{k} \geq 317$ then $k \geq 66$. So for $k \geq 66$ the inequality (2) equivalent to $U_{k}<x_{0}$, where

$$
U_{k}=\frac{4 \log p_{k}-\log 4}{\log \log p_{k}+\beta-\frac{1}{2 \log ^{2} p_{k}}+\frac{1}{p_{k}}-2}-\log 2 .
$$

Let $k_{1}=373707$, then for $y \geq \log \left(p_{k_{1}}\right)$, the function

$$
y \longmapsto f(y)=\frac{4 y-\log 4}{\log y+\beta-\frac{1}{2 y^{2}}+\frac{1}{e^{y}}-2}-\log 2,
$$

is strictly increasing, because for $y \geq \log \left(p_{k_{1}}\right)$, we have $-6+2 \beta+2 \ln y>0$ and

$$
f^{\prime}(y)=8 y^{2} \frac{e^{y}\left(\ln 2 \frac{e^{y}}{y}+(-6+2 \ln y+2 \beta) y^{2} e^{y}+(2-\ln 2) y^{2}+2 y^{3}+(y \ln 2-3) e^{y}\right)}{\left(2 y^{2}-4 y^{2} e^{y}-e^{y}+2 y^{2} e^{y} \ln y+2 y^{2} \beta e^{y}\right)^{2}}>0 .
$$

So the sequence $\left(U_{k}\right)_{k \geq 373707}$ is strictly increasing, then we get $\min _{k \geq 66} U_{k}=\min _{66 \leq k \leq 373707} U_{k}$. A computer calculation gives $\min _{k \geq 66} U_{k} \simeq 59.9$, then $x_{0}=60$. Let us choose $k_{0}$ to be the smallest integer such that $U_{k}<60$. A computer calculation gives $k_{0}=130947$ and $p_{k_{0}}=1740611$. So, if we take $\mathcal{A}=\mathcal{B}_{2}^{k_{0}}$, we get $S\left(\mathcal{B}_{2}^{k_{0}}, x\right)>S(\mathcal{P}, x)$ for $x \geq x_{0}$. According to Lemma 2.4, we have:

$$
S\left(\mathcal{B}_{2}^{k}, x\right)>S\left(\mathcal{B}_{2}^{k_{0}}, x\right)>S(\mathcal{P}, x) \text { for } k \geq k_{0} \text { and } x \geq x_{0} .
$$

This completes the proof. 
Remark 1. By Lemma 2.4, we can ask the following question. Is it true that for $x>0$, there exists $k$ such that $S\left(\mathcal{B}_{2}^{k}, x\right)>S(\mathcal{P}, x)$.

\section{References}

[1] Clark, D. A. (1995). An upper bound of $\sum 1 /\left(a_{i} \log a_{i}\right)$ for primitive sequences, Proceedings of the American Mathematical Society, 123, 363-365.

[2] Cohen, H. (1991). High precision computation of Hardy-Littlewood constants. Preprint. Available online at: http://www.math.u-bordeauxl.fr/ cohen/hardylw. dvi

[3] Erdôs, P. (1935). Note on sequences of integers no one of which is divisible by any other. Journal of the London Mathematical Society, 10(2), 126-128.

[4] Erdős, P. (1988). Seminar at the University of Limoges.

[5] Erdős, P., \& Zhang, Z. (1993). Upper bound of $\sum 1 /\left(a_{i} \log a_{i}\right)$ for primitive sequences. Proceedings of the American Mathematical Society, 117(4), 891-895.

[6] Farhi, B. (2017). Results and conjectures related to a conjecture of Erdós concerning primitive sequences. Preprint. arXiv:1709.08708v2 [math.NT].

[7] Laib, I., Derbal, A., \& Mechik, R. (2019). Somme translatée sur des suites primitives et la conjecture d'Erdős. Comptes Rendus Mathematique, 357(5), 413-417.

[8] Lichtman, J. D., \& Pomerance, C. (2019). The Erdős conjecture for primitive sets. Proceedings of the American Mathematical Society Ser. B, 6, 1-14.

[9] Rezzoug, N., Laib, I., \& Guenda, K. (2020). On a translated sum over primitive sequences related to a conjecture of Erdős. Notes on Number Theory and Discrete Mathematics, 26(4), $68-73$.

[10] Rosser, J. B., \& Schoenfeld, L. (1962). Approximates Formulas for Some Functions of Prime Numbers. Illinois Journal of Mathematics, 6, 64-94.

[11] Zhang, Z. (1991). On a conjecture of Erdős on the sum $\sum_{p \leq n} 1 /(p \log p)$. Journal of Number Theory, 39, 14-17.

[12] Zhang, Z. (1993). On a problem of Erdős concerning primitive sequences. Mathematics of Computation, 60(202), 827-834. 